\title{
FIB TEM Cross-section Sample Preparation of Thin Metal Films Deposited on Polymer Substrates.
}

\author{
F. Rivera, J. Abbott, R.C. Davis, and R. Vanfleet \\ Department of Physics and Astronomy, Brigham Young University, Provo, UT 84602
}

The integration of electronic components onto flexible substrates has created many interesting products, from flexible displays and solar cells to wearable electronics. This, in conjunction with increasingly shrinking electronic geometries, has opened the need to study the behavior of these materials and devices in the nano scale, in a manner parallel to semiconductor manufacturing. Traditionally, semiconductor device manufacturers rely on cross-sectional Transmission Electron Microscopy (TEM) for studying and monitoring the process, observing and investigating defects, as well as interface layers. Furthermore, Focused Ion Beam (FIB) TEM sample preparation has also provided a tool to produce high-quality, site-specific, TEM cross-section samples for the semiconductor industry. Just like in the semiconductor industry, sample preparation is one of the critical steps in TEM analysis that significantly determines the quality characterization and chemical analysis of the smallest and most critical structures. However, the use of polymers as the substrate presents an array of new challenges that must be overcome in order to apply the techniques used for semiconductors for the study of these devices. For example, due to the nature of the substrate, as the sample is thinned to a thickness suitable for TEM analysis, the sample will begin to warp, heat up, charge, and melt.

While some solutions, such as microtomy and cryo-FIB are currently employed to address several of these problems, in this paper we would like to present and add here an alternative FIB cross-sectional TEM sample preparation. As a proof of concept of this method several TEM samples were prepared and analyzed by TEM and EDX, some of these samples included commercial recordable DVDs. The sample presented in this abstract was taken from a thin gold film deposited on a polycarbonate substrate used to manufacture an archival-grade DVD. Figures 1a and 1b show a summarized version of this method, wherein a process similar to making an "h-bar" cross-section sample is used. However, pillars are left for structural support of the polymer substrate, thus preventing the TEM specimen from folding onto itself. Furthermore, Transmission Electron Micrographs, (fig. 1c and 1d) and Energy-Dispersive X-Ray Spectroscopy (EDX) line scans (fig. 2b and 2c) were taken from the sample as a way of determining the quality of the resulting samples. Though there was a concern for significant $\mathrm{Ga}$ ion implantation in the prepared samples (due to their softness), it was observed in several cases that the Ga counts were low to none. Also, despite the softness of various substrates studied, this method yielded viable cross-section TEM samples from polymer substrates. EDX line scans, a common tool for chemical profiling in the semiconductor industry, showed a $<7 \mathrm{~nm}$ spatial resolution for these samples (as measured from peak to valley in the EDX line scan profile).

This approach has the advantage of extending the use of FIB instruments (currently without a cryostage) to prepare site-specific TEM samples for the study of materials deposited onto polymer substrates. 

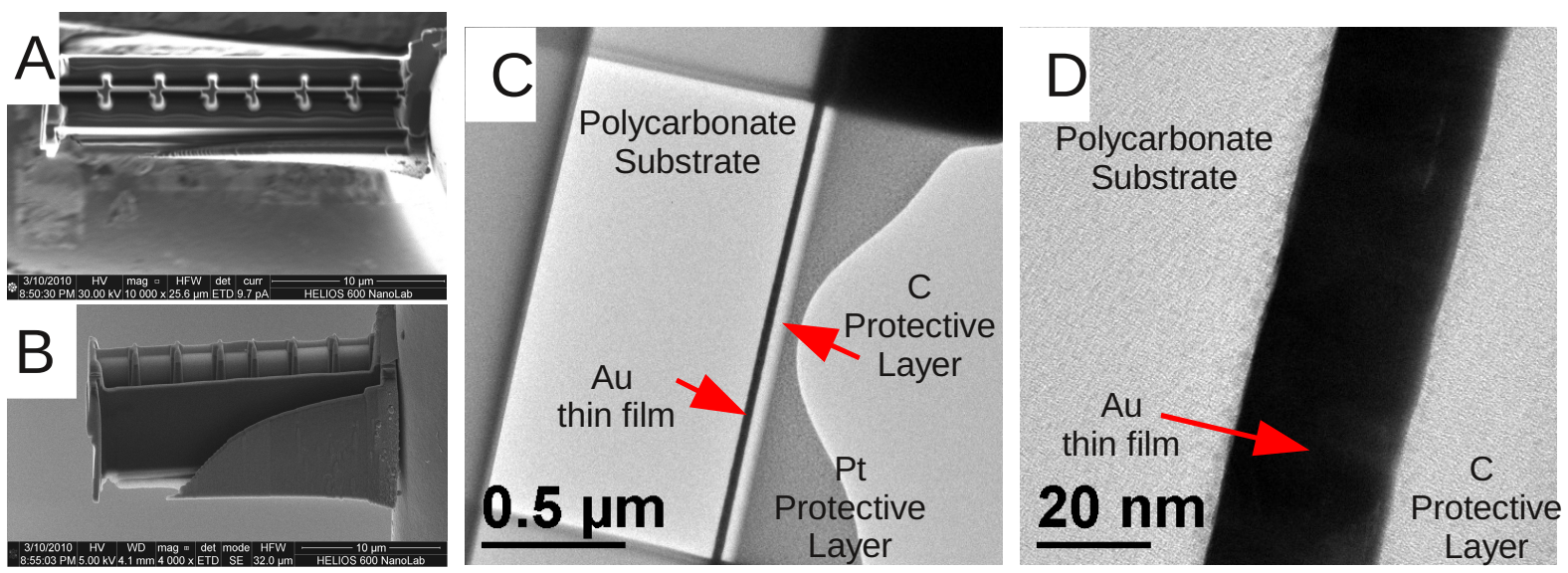

FIG. 1. Top (A) and side (B) views of the modified series of "h-bar" sections, leaving structural supports that prevent the polycarbonate substrate from warping under the focused ion beam. Also, Bright-Field Transmission Electron Micrographs of (C) one of the thin sections between two supports showing the polycarbonate substrate, the $\mathrm{Au}$ thin film, a thin protective $\mathrm{C}$ coating, and a thicker Pt protective layer. (D) A higher magnification of the Au thin film $(\sim 40 \mathrm{~nm})$ thick deposited onto a polycarbonate substrate, showing in better detail the granular structure of the film.

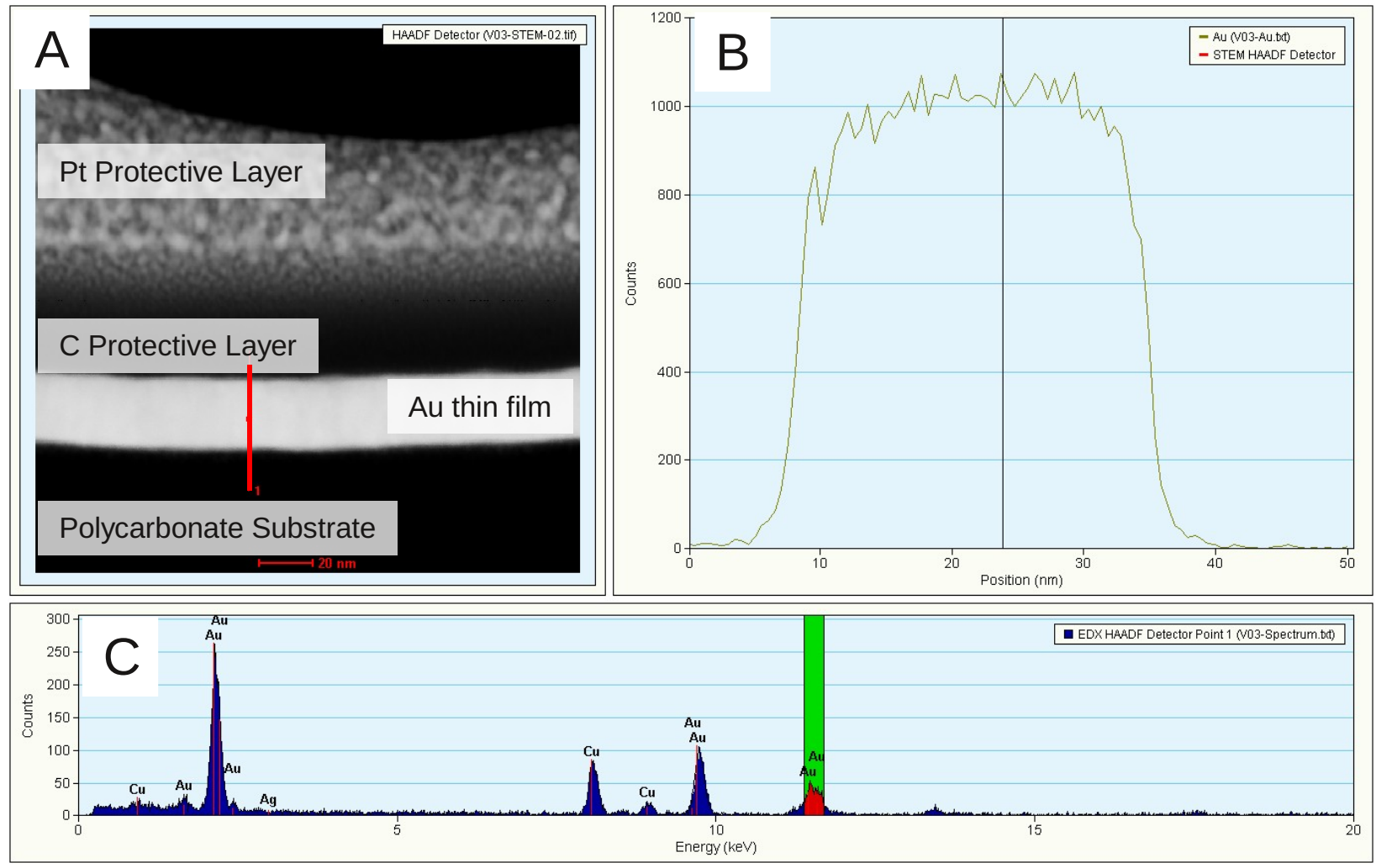

FIG. 2. (A) Scanning Transmission Electron Micrograph of the layers mentioned in fig.2. (B) Energy Dispersive X-Ray Spectroscopy (EDX) line scan of the Au thin film taken along the vertical line shown in (A). (C) EDX spectra taken partway along the Au thin film. Low to none traces of $\mathrm{Ga}$ from the FIB process observed in the spectra. 